\title{
A Boundary Layer Approach in the Modelling Flows in Microscale
}

\author{
Alexander S. Liberson \\ Mechanical Engineering Department \\ Rochester Institute of Technology \\ 76 Lomb Memorial Drive, Rochester, NY 14623-5604, USA \\ asleme@rit.edu
}

\begin{abstract}
In this paper we discuss a gradient model as an extension of classical fluid mechanics with a higher order of spatial and time derivatives of velocities, pressure and acceleration. Size effects, characterized by a mean free path, or the size of a microstructural lattice are captured by this model making feasible its applications in micro and nano-scales. One of the challenges of gradient models is to keep the number of additional constitutive parameters to a minimum. In contrast to the number of gradient models, requiring a large quantity of phenomenological constants, the present model requires a one additional constant, linked to the microscale characteristic length for the steady state flow, and two additional parameters in a non-steady counterpart. In order to account for the micro - structure the higher order continuum approximation is built based on a continualization strategy inside microstructural volume and a relating variational principle. This model does not require introduction of a gyration vector, or coupled stresses, although these quantities could be deduced by coordinating with the micropolar or another gradient theory. The derived model exactly matches the non-steady linearized micropolar fluid flow model (small gyrations and velocity distributions), giving analytical interpretation of typical micropolar empirical coefficients in terms of a Knudsen number and a time characteristic constant.

An asymptotic behaviour of a two - dimensional flow through a microchannel is investigated via rigorous singular perturbation analysis with respect to the small parameter, proportional to the Knudsen number. This small parameter is involved in both partial differential equations and the Maxwell's slip boundary conditions. A singular perturbation technique reveals a thin boundary layer (Knudsen layer) region near the solid boundary described by the inner solution, whereas the outer solution relates to the classical NavierStokes model. For the boundary layer solution is presented in an analytical form, whilst the outer region is modelling using analytical solution in case of a canonical, or a numerical solution for a non-canonical domain respectfully. Calculational results obtained compare favourably with the currently available experimental data.
\end{abstract}

Keywords: Microscale, gradient model, Knudsen boundary layer, asymptotic analysis.

\section{Nomenclature}

$\begin{array}{cl}\mu & \text { dynamic viscosity } \\ \varrho & \text { fluid density } \\ \mathrm{Kn} & \text { Knudsen number } \\ L & \text { hydraulic diameter } \\ 1 & \text { characteristic length in a microscale } \\ p^{\prime} & \text { pressure gradient } \\ \mathrm{u} & \text { axial velocity } \\ (\mathrm{x}, \mathrm{y}) & \text { Cartesian coordinates } \\ \Delta & \text { Laplace operator } \\ c_{p} & \text { Maxwell's slip coefficient } \\ & \text { Subscripts: } \\ (x, y) & \text { partial derivatives by relating coordinates }\end{array}$




\section{Introduction}

When mechanical, thermal, or electrical device is decreased in size to a sufficiently small level, its characteristics become significantly different from their counterparts in a macroscale. Microscale effects become important, when the mean free path of the energy carrier becomes comparable to the characteristic length of the object. In such scale the continuum approach based on a heuristic principle of continuity is no longer valid. Different models of a non-classical mechanics and physics have been introduced using different names for the generalized continuum: Cosserat, gradient, nonlocal, nonsymmetric, microstructure, micropolar, couple stress, multipolar, micromorphic, multiscale and others. A survey of different non-classical theories can be found in [1-6]. All these models include the higher order derivatives in mathematical formulations and additional empirical constants, whose experimental verification meets certain difficulties. The higher order gradients introduce additional degrees of freedom allowing to continualize the discrete matter, representing better as a result the discrete microstructure. The higher order gradients provide also a regularization and smoothing of nonphysical singularities and discontinuities predicted by classical models of continuum.

It is agreeable to differentiate flow conditions in microchannels based on a rarefaction effect characterized by the Knudsen number. In the free molecular flow conditions $(\mathrm{Kn}>10)$ the Boltzmann equations are solved using typical approaches as the lattice Boltzmann model, direct simulation Monte-Carlo method, molecular dynamics. A comprehensive review of relating approaches could be found in [7,8]. Within the Knudsen number range $0.1<\mathrm{Kn}<10$ a rarefied gas can be considered as a continuum - transition flow, which is neither a continuous medium nor a free -molecular flow. To model these flows, a number of generalized hydrodynamics models have been proposed. The family of Burnett models replaces Boltzmann kinetic equation by series of differential equations in a reduced space with respect to the finite number of moments of kinetic function distribution. However, attempts at solving the Burnett equations have uncovered many physical and numerical difficulties produced by the model. The trace of evolution of Burnett models and description of the progress made by recent developments could be found in [9].

Within the range of $0.001<\mathrm{Kn}<0.1$ the micro fluid flow is often characterized as a slip flow [7, 8, 10]. Typical engineering analyses of fluid flows in microchannels and micromachined fluid systems are based on a simplified approach accounting for the slip flow at the boundary within the frame of classical Navier-Stokes model. However, slip boundary conditions are not the only intrinsic property of a microscale. Physical phenomena arousing in microscale is associated with the microstructure and microrotation of fluid molecules, which should be accounted by the model to present an adequate description of microfluidics. The monographs $[2,4]$ provide a unified picture of relating mathematical theory based on a micropolar model. A number of publications indicate that flow analysis in microchannels, based on a gradient theory of micropolar fluids gives better predictions of experimental results [11-13]. Additionally, we note that the presence of roughness may prevent flow from the slip at the solid boundary [14]. In this case application of a simplified engineering microfluidic theory [10] completely ignores microstructure making no difference to the application of the classical NavierStokes model to microscale. On a contrary, gradient models result in a correction for classical macro fluid predictions of a flow rate, average velocity, Darcy friction factor and pressure distribution regardless of a roughness effect on a boundary conditions at microscale.

The demarcation line between application to microflows of gradient models versus classical Navier-Stokes model, supplemented by slip conditions, is not clearly defined. In $[15,16]$ the comparative results are presented for a one dimensional case of a pressure driven flow for the wide range of Knudsen numbers. The present work extends application of the developed gradient model to the two-dimensional case with a following comparison of both approaches. An asymptotic behaviour of a two - dimensional flow through a microchannel is investigated via rigorous singular perturbation analysis with respect to the small parameter, proportional to the Knudsen number. This small parameter is involved in both partial differential equations and the Maxwell's slip boundary conditions. A singular perturbation technique reveals a thin boundary layer (Knudsen layer) region near the solid boundary as an inner solution, whereas the outer solution satisfies to the classical Navier-Stokes equations. The boundary layer solution is presented in an analytical form, whereas the outer region is modelled using analytical or numerical solutions for the canonical or non-canonical domains respectfully. Calculational results obtained compare favourably with the currently available experimental data. 


\section{Fluid flow in microscale}

Consider pressure driven steady state isothermal flow in a cylindrical microchannel of an arbitrary shape. The governing equation expresses the momentum balance, which is in a stationery condition reads [14] ( $u$ - axial component of the flow velocity)

$$
\begin{gathered}
\varepsilon^{2} \Delta \mathrm{v}-\mathrm{v}+\frac{p^{\prime}}{\mu}=0, \\
\Delta \mathrm{u}=\mathrm{v}, \quad \varepsilon=\frac{l}{\sqrt{6}}=K n \frac{\mathrm{L}}{\sqrt{6}}
\end{gathered}
$$

With application to the rectangular cross section $-a<x<a,-b<y<b$, the low order boundary conditions are:

$$
\begin{gathered}
\left.u_{x}\right|_{x=0}=0,\left.\quad u_{y}\right|_{y=0}=0 \\
\left.\left(u+\varepsilon_{1} \frac{\partial u}{\partial x}\right)\right|_{x=a}=0,\left.\quad\left(u+\varepsilon_{1} \frac{\partial u}{\partial y}\right)\right|_{y=b}=0, \quad \varepsilon_{1}=\sigma_{p} l=K n * \sigma_{p} L
\end{gathered}
$$

whilst the higher order boundary layer related conditions, read

$$
\begin{array}{rlrl}
\left.\mathrm{v}_{x}\right|_{x=0} & =0, & & \left.\mathrm{v} y\right|_{y=0}=0 \\
\left.\mathrm{v}\right|_{x=a}=0, & \left.\mathrm{v}\right|_{y=b}=0
\end{array}
$$

Conditions (2a) are the symmetry conditions, and (2b) are the $1^{\text {st }}$ order Maxwell slip boundary conditions at the liquid -solid interphase. The additional higher order conditions pertain to symmetry at $x=0$ and $y=0,(3 \mathrm{a})$, and to the boundary layer conditions (3b) at the solid liquid interphase.

The difficulty of a multidimensional problem (1)-(3) precludes from solving it exactly. However, the presence of a small parameter (squared Knudsen number) indicates existence of a thin boundary layer region, opening up a possibility to use a simplified model. The basic tool explored in this section is similar to the one used in Prandtl's boundary layer theory, where the flow around an airfoil is treated as inviscid far away from the wall, but viscous in its proximity. The present approach identifies the core flow by using classical Navier-Stokes model with the following boundary layer correction in a close vicinity to the wall.

A two - scale asymptotic expansion is defined as the following

$$
\mathrm{v}(x, y)=\frac{p^{\prime}}{\mu}+U\left(\frac{x-a}{\varepsilon}, y\right)+V\left(x, \frac{y-b}{\varepsilon}\right)
$$

where the boundary layers coordinates are introduced $X=\frac{x-a}{\varepsilon}, Y=\frac{y-b}{\varepsilon}$ in the vicinities to the edges $x=a$ and $y=b$ accordingly. Introducing (4) into (1a) obtain

$$
\varepsilon^{2} \Delta \mathrm{v}-\mathrm{v}+\frac{p^{\prime}}{\mu}=\left(U_{X X}+\varepsilon^{2} V_{Y Y}\right)+\left(\varepsilon^{2} U_{X X}+V_{Y Y}\right)-\left(\frac{p^{\prime}}{\mu}+U+V\right)+\frac{p^{\prime}}{\mu}=0
$$

The leading order equations for the boundary layers

$$
U_{X X}-U=0, \quad V_{Y Y}-V=0
$$


identify solution exponentially decaying away from the walls, $U \sim e^{\frac{x-a}{\varepsilon}}, V \sim e^{\frac{y-b}{\varepsilon}}$. As an outcome, the leading order solution (39) satisfying boundary conditions (38) reads

$$
\mathrm{v}(x, y)=\frac{p^{\prime}}{\mu}\left(1-e^{\frac{x-a}{\varepsilon}}-e^{\frac{y-b}{\varepsilon}}\right)
$$

The presented asymptotic solution (42) is valid under the assumption that the boundary of a domain is a smooth curve. In case of a boundary, contained corner points, the solution (42) should be supplemented by the corner boundary function [23], dying rapidly away from the point $x=a, y=b$. Considering geometry where the area, relating to the corner boundary layer is negligible compared to the total area occupied by the edge boundary layer, the local corner effect can be neglected.

To find velocity distribution $\mathrm{u}(x, y)$ consider the Poisson equation (36b). We are looking for the solution as an expansion by a complete set of eigenfunctions using the generalized double Fourie series

$$
u(x, y)=\sum_{m=1}^{\infty} \sum_{n=1}^{\infty} A_{m n} \varphi_{m}(x) \psi_{n}(y)
$$

where

$$
\begin{array}{cl}
\varphi_{m, x x}-\varphi_{m}=0, & \varphi_{m, x}(0)=\varphi_{m}(a)+\varepsilon_{1} \varphi_{m, x}(a)=0 \\
\psi_{n, y y}-\psi_{n}=0, & \psi_{n, y}(0)=\psi_{n}(b)+\varepsilon_{1} \psi_{n, y}(b)=0
\end{array}
$$

The solution of (9) yields $\varphi_{m}(x)=\cos \left(\lambda_{m} x\right), \psi_{n}(y)=\cos \left(\lambda_{n} y\right)$, satisfying slip boundary conditions, where the eigenvalues are the roots of the following transcendental equations

$$
\tan \left(\lambda_{m} a\right)=\left(\varepsilon_{1} \lambda_{m}\right)^{-1}, \quad \tan \left(\lambda_{n} b\right)=\left(\varepsilon_{1} \lambda_{n}\right)^{-1}
$$

To find unknown coefficients $A_{m n}$, we plug expansion (43) into the equation (36b), forming inner product with each of the elements of the set $\left\{\varphi_{m}(x) \psi_{n}(y)\right\}$. Carrying out the leading order approximation terms, arrive

$$
\begin{gathered}
A_{m n}=-\frac{4 p^{\prime}}{\mu \overline{a b}} \frac{\sin \left(\lambda_{m} a\right) \sin \left(\lambda_{n} b\right)}{\lambda_{m} \lambda_{n}\left(\lambda_{m}^{2}+\lambda_{n}^{2}\right)}\left[1-\varepsilon^{2}\left(\lambda_{m}^{2}+\lambda_{n}^{2}\right)\right]+O\left(\varepsilon^{4}\right) \\
\bar{a}=a+\frac{\sin \left(2 \lambda_{m} a\right)}{2 \lambda_{m}}, \quad \bar{b}=b+\frac{\sin \left(2 \lambda_{n} b\right)}{2 \lambda_{n}}
\end{gathered}
$$

The presented solution (8), (11) is a superposition of the classical Poiseuille solution, following from the above at $\varepsilon=$ $\varepsilon_{1}=0$, and its correction, affected by the Knudsen boundary layer. We can simplify the procedure of finding the roots of transcendental equations (10) by the substituting (10) with its asymptotic expansion in terms of a small parameter $\varepsilon_{1}$. When $\varepsilon_{1}=0$ (10) reduces to $\tan \left(\lambda_{m} a\right)=\infty, \tan \left(\lambda_{n} b\right)=\infty$, whose roots are $\lambda_{m 0} a=(m-0.5) \pi, \lambda_{n 0} a=(n-0.5) \pi$. If $\varepsilon_{1}$ is small, but finite, we expect roots $\lambda_{m}$ and $\lambda_{n}$ to deviate slightly from $\lambda_{m 0}$ and $\lambda_{n 0}$. In this case we assume that the roots have an expansion $\lambda_{m}=\lambda_{m 0}+\varepsilon_{1} \Delta \lambda_{m}+\cdots, \quad \lambda_{n}=\lambda_{n 0}+\varepsilon_{1} \Delta \lambda_{n}+\cdots$. By substituting the assumed expansion into the transcendental equation (45), and neglecting all quadratic and higher order terms of $\varepsilon_{1}$, obtain 


$$
\lambda_{m}=\left(1-\varepsilon_{1}\right) \frac{(2 m-1) \pi}{2 a}, \quad \lambda_{n}=\left(1-\varepsilon_{1}\right) \frac{(2 n-1) \pi}{2 b}
$$

\section{Results}

Two- dimensional solutions in Cartesian coordinates were obtained using trigonometric expansion (8) with the coefficients calculated according to (11). It was sufficient to use 10 terms of the expansion (8) in each direction due to its fast convergence. The first ten eigenvalues $\lambda_{m} a$, obtained by solving the transcendental equation (45) for $K n=0.1$ are presented in the table below

Table 1: Eigenvalues of equations (10) calculated for $K n=0.1$.

\begin{tabular}{|c|c|c|c|c|c|c|c|c|c|c|}
\hline $\mathbf{m}$ & 1 & 2 & 3 & 4 & 5 & 6 & 7 & 8 & 9 & 10 \\
\hline$\lambda_{m} a$ & 1.404 & 4.241 & 7.145 & 10.113 & 13.131 & 16.1835 & 19.257 & 22.348 & 25.449 & 28.558 \\
\hline
\end{tabular}

The experimental data from [11] along with predictions from the Poiseuille model gradient models for the channel 3000 $\times 600 \times 30 \mu m^{3}(L \times W \times H)$ are presented In Figure 1. Square markers designate the testing data as the average data of three consecutive experimental runs. The gradient model at $K n=0.1$ (solid line) predicts the experimental data better than the classical Navier-Stokes theory (dash line).

Figure 2 shows the experimental data from [11] compared to the predictions by the Navier-Stokes theory, micropolar and gradient models. The channel used to obtain experimental data, marked by squares, is $3000 \times 600 \times 30 \mu \mathrm{m}^{3}$. Microchannels used to compare to micropolar theory are $10,000 \times 60 \times 25.4 \mu^{3}$, and 11,700 $\times 80 \times 20 \mu^{3}$. The gradient model provides a good prediction of experimental data over the wide range of applied pressure drop and a flow range.

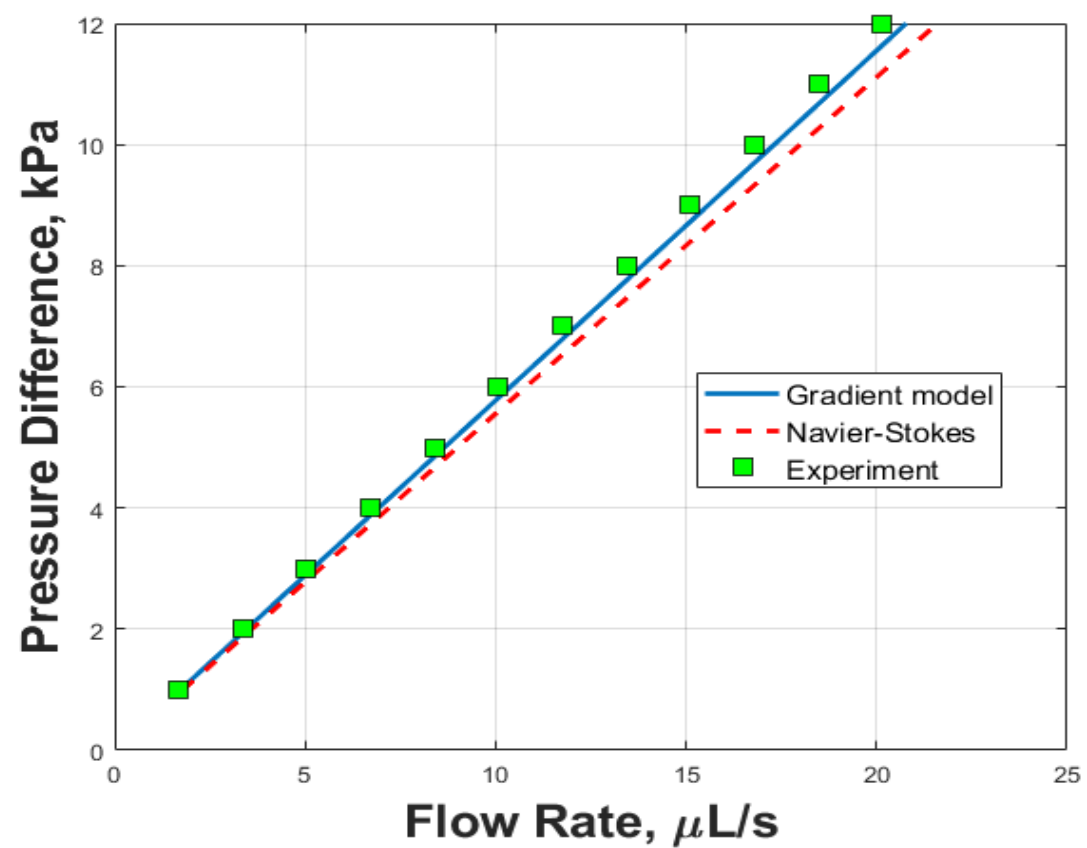

Fig. 1: Comparison of the Navier-Stokes model, gradient model and the experimental data for water flow in the microchannel $3000 \times 600 \times 30 \mu \mathrm{m}^{3}(\mathrm{~L} \times W \times \mathrm{H})$. 


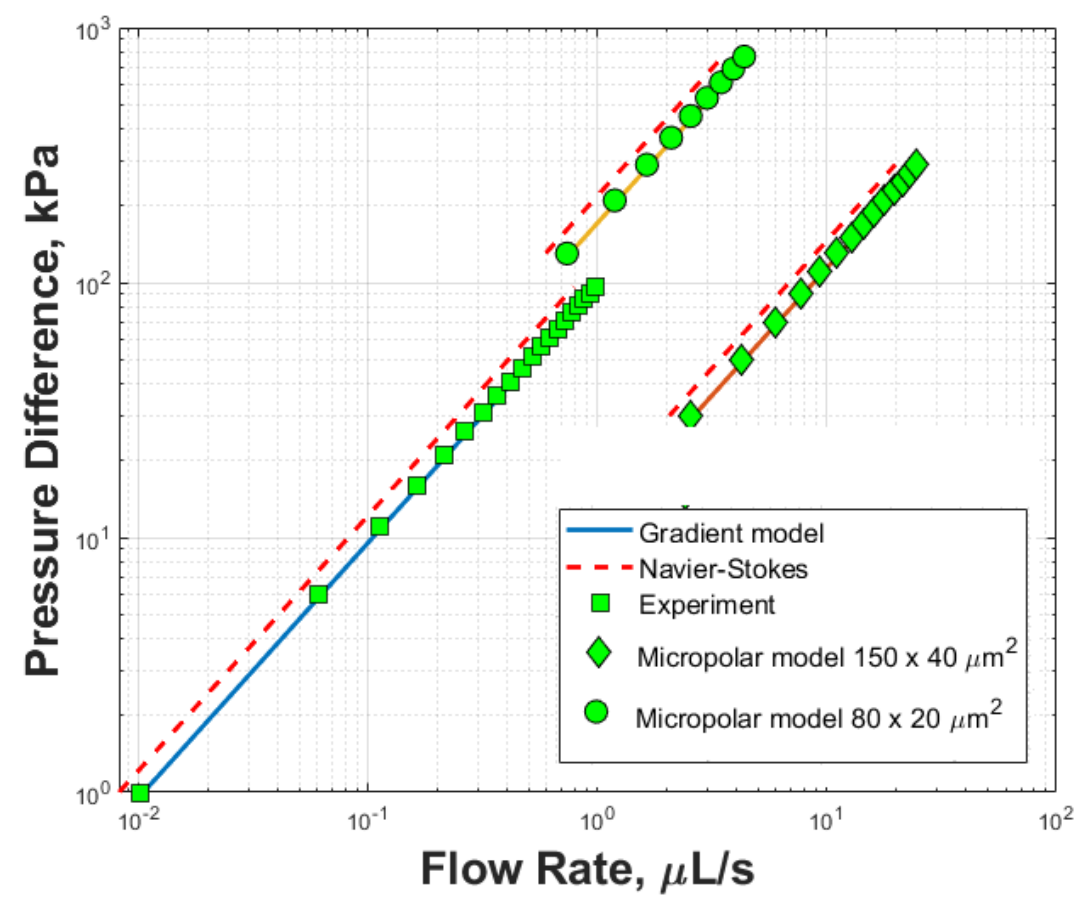

Fig. 2: Comparison of the experimental data with the Navier-Stokes model, gradient model and the micropolar model for water flow in three microchannels.

\section{Conclusion}

An asymptotic behaviour of a two - dimensional flow through a microchannel is investigated via rigorous singular perturbation analysis with respect to the small parameter, proportional to the Knudsen number. A singular perturbation technique reveals a thin boundary layer (Knudsen layer) region near the solid boundary described by the inner solution, whereas the outer solution relates to the classical Navier-Stokes model. Calculational results obtained compare favourably with the currently available experimental data.

\section{References}

[1] H. Askes, A. Suiker, L.Sluys, "A classification of higher order strain-gradient models-linear analysis," Archive of Applied Mechanics, vol. 72, no. 2-3, pp. 171-188, 2002.

[2] A. S. Eringen, Micro Continuum Field Theories II: Fluent Media. New York: Springer Verlag, 2001.

[3] A. S. Eringen, "Micropolar Theory of Liquid Crystals," In: J. F. Johnson and R. S. Porter, Eds., Liquid Crystals and Ordered Fluids, vol. 3, New York: Plenum Publishing, New York, 1978.

[4] G. Lukaszewicz, "Micropolar Fluid Theory and Applications," Modeling and Simulation in Science, Engineering and Technology, Boston, Mass, USA: Birkhauser, 1999.

[5] P. Power, "Bio-Fluid Mechanics," Advances in Fluid Mechanics, UK: W.I.T. Press, 1995.

[6] V. K. Stokes. "Theories of Fluids with Microstructure," Berlin, Springer-Verlag, 1984.

[7] G. Karniadakis, A. Beskok, N. Alurtu Microflows and Nanoflows. Fundamentals and Simulation. Springer, 2000.

[8] Y. Song, D. Cheng, L. Zhao, "Microfluidics: Fundamentals, Devices and Applications: Fundamentals and Applications," Wiley-VCH Verlag, 2018.

[9] R. K. Agarwal, R. Balakrishnan, "Beyond Navier-Stokes: Burnett equations for flows in the continuum - transition regime," Physics of Fluids, vol. 14, pp. 1818, 2002. 
[10] S. Kandlikar, S. Garimella, D. Li, S. Colin, M. King, "Heat Transfer and Fluid Flow in Minichannels and Microchannels," Elsevier Ltd, 2014.

[11] I. Papautsky, J. Brazzle, T. Ameel, A. B. Frazier. "Laminar fluid behaviour in microchannels using micropolar fluid theory," Sensors Actuators, pp. 101-108, 1999.

[12] I. Papautsky, J. Brazzle, T. Ameel, A.B. Frazier, "Microchannel fluid behaviour using micropolar fluid Theory," Proc. EMBS 97, Microelectromech. Sys., Chicago, 1997, pp. 2285-2291.

[13] Y. Zhu, and S. Granick, "Limits of the hydrodynamic no-slip boundary condition," Phys. Rev. Lett., vol. 88, no. 10, pp. 106102, 2002.

[14] A. S. Liberson, "Microscale: wave propagation modelling using a high-order gradient continuum Theory", in Dekker Encyclopedia of Nanoscience and Nanotechnology, E. Lyshevsky, Ed. Third Ed, vol. 7, 2017.

[15] A. S. Liberson, Y. S. Vahedein, "Variant of a gradient continuum mechanics with application to flow in microchannels", in Proceedings of the 4th International Conference of Fluid Flow, Heat and Mass Transfer (FFHMT'17), Toronto, Canada, 2017.

[16] H. Schlichting. Boundary Layer Theory. John Wiley \& Sons, 2011.

[18] G. A. Korn, T. A. Korn, Mathematical Handbook for Scientists and Engineers. McGraw-Hill, 1968.

[19] C. K. Kang, A. S. Eringen, "The effect of microstructure on the rheological properties of blood," Bull. Math. Biol,. vol. 38, pp.135-158, 1976.

[20] G. C. Hazanka, B. Phukan, "Effects of variable viscosity and thermal conductivity on MHD flow of micropolar fluid in a continuous moving flat plate," International Journal of Computer Applications, vol. 122, pp. 8, 2015.

[21] Z. Crnyaric, N. Mujacovic, "Numerical analysis of the solutions for 1D compressible viscous micropolar fluid flow with different boundary conditions," Mathematics and Computers in Simulation, vol. 126, pp. 45 - 62, 2016.

[22] S. Richardson, "On the no-slip boundary conditions", J. Fluid Mech., vol. 58, pp. 707-719, 1973.

[23] G. Mo, F. Rosenberger, "Molecular dynamics simulation of flow in a two dimensional channel with atomically rough walls," Phys. Rev. A, vol. 42, pp. 4688-4692, 1990.

[24] H. Han, R. Kellogg, "Differentiability properties of solutions of the equation $-\varepsilon^{2} \Delta u+r u=f(x, y)$ in a square," SIAM J. Math. Anal, vol. 21, no. 2, pp. 394-408, 1990. 